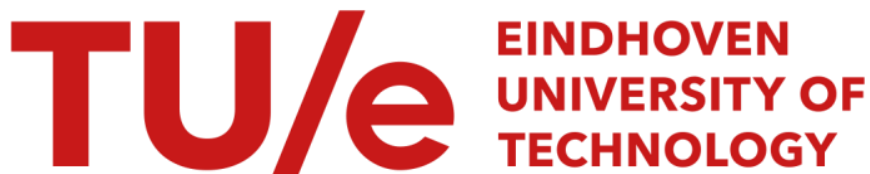

\section{Small scale velocity jumps in shear turbulence}

\section{Citation for published version (APA):}

Staicu, A. D., \& Water, van de, W. (2003). Small scale velocity jumps in shear turbulence. Physical Review Letters, 90(9), 094501-1/4. [094501]. https://doi.org/10.1103/PhysRevLett.90.094501

DOI:

10.1103/PhysRevLett.90.094501

Document status and date:

Published: 01/01/2003

\section{Document Version:}

Publisher's PDF, also known as Version of Record (includes final page, issue and volume numbers)

\section{Please check the document version of this publication:}

- A submitted manuscript is the version of the article upon submission and before peer-review. There can be important differences between the submitted version and the official published version of record. People interested in the research are advised to contact the author for the final version of the publication, or visit the $\mathrm{DOI}$ to the publisher's website.

- The final author version and the galley proof are versions of the publication after peer review.

- The final published version features the final layout of the paper including the volume, issue and page numbers.

Link to publication

\section{General rights}

Copyright and moral rights for the publications made accessible in the public portal are retained by the authors and/or other copyright owners and it is a condition of accessing publications that users recognise and abide by the legal requirements associated with these rights.

- Users may download and print one copy of any publication from the public portal for the purpose of private study or research.

- You may not further distribute the material or use it for any profit-making activity or commercial gain

- You may freely distribute the URL identifying the publication in the public portal.

If the publication is distributed under the terms of Article $25 \mathrm{fa}$ of the Dutch Copyright Act, indicated by the "Taverne" license above, please follow below link for the End User Agreement:

www.tue.nl/taverne

Take down policy

If you believe that this document breaches copyright please contact us at:

openaccess@tue.nl

providing details and we will investigate your claim. 


\title{
Small Scale Velocity Jumps in Shear Turbulence
}

\author{
Adrian Staicu and Willem van de Water \\ Physics Department, Eindhoven University of Technology, Postbus 513, 5600 MB Eindhoven, The Netherlands
}

(Received 29 August 2002; published 3 March 2003)

\begin{abstract}
We measure structure functions and structures in uniformly sheared strong turbulence using an array of hot-wire velocity sensors. We find that the large-scale shear persists down to the smallest scales. There is a marked asymmetry between velocity increments measured in the shear direction, and those measured in the plane perpendicular to it. In the shear direction the scaling exponents tend to a constant, signifying the presence of small-scale cliffs. Direct evidence for those is presented by the spatial structure of the strongest velocity gradients.
\end{abstract}

DOI: 10.1103/PhysRevLett.90.094501

Fluid turbulence involves a wide range of scales. Motion on large scales will carry the imprint of anisotropic forcing, but according to Kolmogorov theory [1], the small-scale eddies will have lost memory of the way in which the large scales are stirred. This return to isotropy underlies the construction of turbulence models which are needed for the computation of turbulent flows in cases of practical interest. A startling recent discovery is that isotropy will probably never be restored, not even at very large Reynolds numbers where very small scales are reached [2,3]. In numerical simulations at low Reynolds numbers, the failure of return to isotropy has been linked to vortex sheets [4] which have been rolled up by the shear. In this Letter we will for the first time show that these structures can indeed be observed in an experiment, and we will relate them to the anisotropic scaling properties of the velocity field.

The test case is homogeneous shear turbulence, which has a linear variation of the mean flow velocity $U$ in the shear (here $y$ ) direction, a constant fluctuation velocity $u$, and an energy spectrum that does not depend on $y$. It is the simplest possible anisotropic turbulent flow, whose large-scale anisotropy is characterized by a single number: the shear rate $S=d U / d y$. Creating such a flow is an experimental challenge, as is a measurement of the statistics of velocity increments over separations in the shear direction $y$ which necessitates the usage of arrays of precisely calibrated velocity probes.

The experimental arrangement is sketched in Fig. 1. With the mean flow $U(y)$ in the $x$ direction, the shear points in the transverse $y$ direction. In the case of homogeneous isotropic turbulence, all odd-order moments, $p=3,5,7, \ldots$, of transverse velocity increments

$$
G_{p}^{T}(r)=\left\langle\left(\Delta^{T} u(r)\right)^{p}\right\rangle=\left\langle\left[u\left(\boldsymbol{x}+\boldsymbol{r} \boldsymbol{e}_{y}\right)-u(\boldsymbol{x})\right]^{p}\right\rangle,
$$

vanish identically because of reflection symmetry. In Eq. (1), $\boldsymbol{e}_{y}$ is the unit $y$ vector and $u$ is the $x$ component of the fluctuating velocity $\left(G_{1}^{T}=0\right)$. In the presence of shear, these odd-order moments will be nonzero at large length scales, but should vanish at small enough $r$ when the isotropy is restored. Down at the smallest scale (the Kolmogorov scale $\eta$ ), the velocity difference in Eq. (1)
PACS numbers: 47.27.Ak, 47.27.Gs, 47.27.Nz

approximates the velocity derivative, and the anisotropy can be quantified through the skewness which is defined as $S_{p}^{T}=\left\langle(\partial u / \partial y)^{p}\right\rangle /\left\langle(\partial u / \partial y)^{2}\right\rangle^{p / 2}, p=3$. Restoration of isotropy implies that the skewness vanishes at large Reynolds numbers. Assuming that, to first order, both the oddorder moments [Eq. (1)] and the skewness are poportional to the shear rate $S$, a simple dimensional argument predicts $S_{3}^{T}$ to decrease with Reynolds number as $S_{3}^{T} \propto \operatorname{Re}_{\lambda}^{-1}$, where $\operatorname{Re}_{\lambda}$ is the Taylor microscale Reynolds number (the Taylor scale is an intrinsic correlation length of turbulence) [5]. Instead, a much slower decay of $S_{3}^{T}$ with $\operatorname{Re}_{\lambda}$ was found in [2] with Reynolds numbers increasing up to $\operatorname{Re}_{\lambda} \approx 10^{3}$. Moreover, the skewness with $p=5$ remained constant, and with $p=7$ it increased with $\operatorname{Re}_{\lambda}$. At smaller Reynolds numbers, such slower decay for $p=3$ was also found in [3], while for $p=5$ it showed a slight decline.

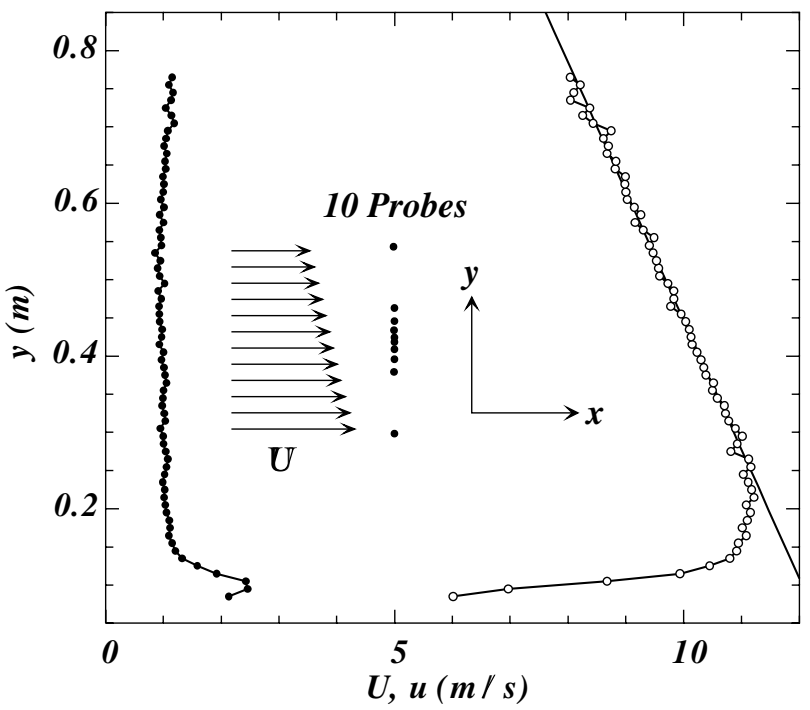

FIG. 1. Homogeneous shear turbulence. Open circles: mean velocity $U$; closed circles: rms fluctuations $u$ at $x / H=5.1$ behind the shear generating grid, where $H=0.9 \mathrm{~m}$ is the height of the tunnel. Near the lower wall the turbulent boundary layer marks the end of the homogeneous shear region. The Reynolds number is $\operatorname{Re}_{\lambda}=630$, and the shear strength $d U / d y=5.95 \mathrm{~s}^{-1}$. 
In this Letter we will concentrate on the structure functions Eq. (1) for which the dimensional argument predicts

$$
G_{p}^{T}(r) \propto S r^{(p+2) / 3}, \quad p=3,5, \ldots
$$

We will show that the properties of the shear are reflected directly in the low-order moments, whereas the high-order moments are determined by very strongly intermittent events, which will be demonstrated to be of a special kind.

We produce homogeneous shear turbulence in a $0.9 \times$ $0.7 \mathrm{~m}^{2}$ cross section recirculating wind tunnel with a maximal Reynolds number $\operatorname{Re}_{\lambda}=630$. To generate a uniform mean velocity gradient we use a novel grid whose $y$ dependent solidity is tuned to preserve a constant rms profile throughout most of the wind tunnel height. The quality of the flow is illustrated in Fig. 1 which shows that the variation of the rms velocity $\left\langle u^{2}\right\rangle^{1 / 2}$ with $y$ is very small and the shear rate is constant over the extent of the probe array $(0.24 \mathrm{~m})$. Furthermore, the measured energy spectra $E\left(k_{x}, y\right)$ at different $y$ were found to be indistinguishable [6]. A very large number of data points $\left[\mathcal{O}\left(10^{9}\right)\right]$ was collected in experiments that ran many hours while velocities were sampled at $2 \times 10^{4} \mathrm{~Hz}$, which is nearly twice the Kolmogorov frequency $U / \eta=1.1 \times 10^{4} \mathrm{~Hz}$, with $\eta=1.6 \times 10^{-4} \mathrm{~m}$.

A few measured transverse structure functions [Eq. (1)], for both even and odd orders, are shown in Fig. 2. Let us recall that the odd-order ones would all have been zero for isotropic turbulence. The tendency to return to isotropy at small $r / \eta$ is strongest for the loworder $(p=3)$, but decreases for the higher-order moments. It means that the return to isotropy is increasingly

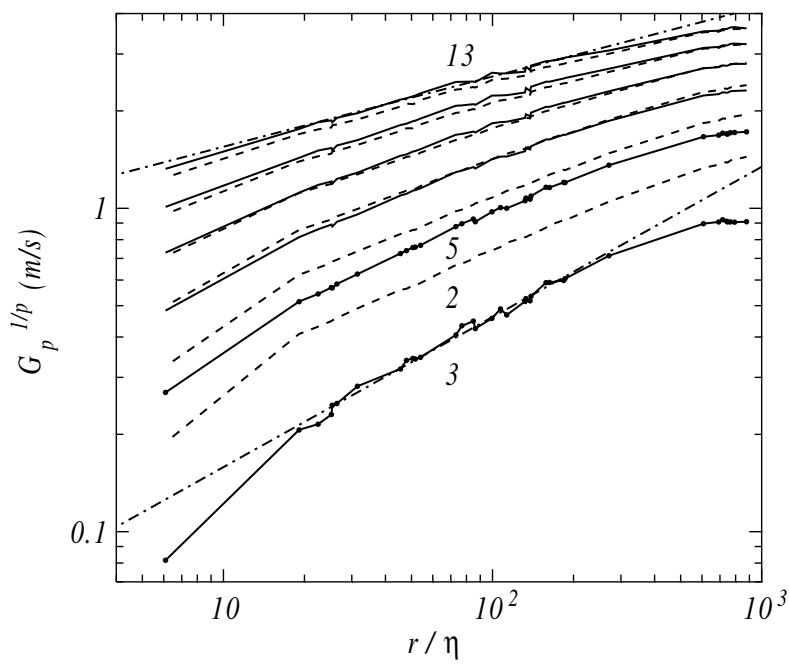

FIG. 2. Structure functions in homogeneous shear turbulence at $\operatorname{Re}_{\lambda}=630$. Full lines: odd orders, $\left|G_{p}\right|^{1 / p}, p=3,5, \ldots, 13$. Dashed lines: even orders, $G_{p}^{1 / p}, p=2,4, \ldots, 12$. Dash-dotted lines: fits of $\left|G_{p}\right| \sim r^{\zeta_{p}}$. The dots in the $p=3,5$ structure functions denote the discrete distances in the probe array. frustrated by the strongest events in turbulence which dominate the high-order structure functions.

The structure functions were measured using a hotwire array of ten probes (sensitive length $200 \mu \mathrm{m}$ ), the 45 distances between them spaced close to exponential, as is illustrated in the inset of Fig. 1. Each point of the structure functions in Fig. 2, therefore, corresponds to a distance $r=y_{i}-y_{j}$ between different probe pairs that are at different locations $y_{i}, y_{j}$. It is seen that the scaling is genuinely in the separation $r$, with the homogeneity of the shear reflected in the smoothness of the curves. The usual measurement [2], with one fixed probe and one probe at different positions $y$, would take a much longer integration time and would have difficulty to discriminate shear inhomogeneity from the $r$ dependence of $G^{T}$.

From the measured structure functions we determined the scaling exponents $\zeta_{p}^{T}$ by fitting $\left|G_{p}^{T}\right| \sim r^{\zeta_{p}^{T}}$. We find a strong asymmetry between odd and even low-order scaling exponents. For example, while $\zeta_{2}^{T} \approx 0.72$ is the value found in homogeneous and isotropic turbulence, $\zeta_{3}^{T} \approx 1.4$, which is close to the value $5 / 3$ predicted by Eq. (2). For the cross spectrum $E_{x y}(k)$ (which vanishes in isotropic turbulence) the equivalent prediction would be $E_{x y}(k) \sim k^{\alpha}$, with $\alpha=-7 / 3$. Consistency with this prediction was concluded in [8] where it was found that $\alpha=-2.1$, while slightly larger $\alpha$ were seen in other experiments [9]. Surprisingly, the conclusions of a recent paper [7] are at odds with these findings, and no asymmetry between even and odd transverse exponent was observed.

From the measured probability density functions (PDF's) $P_{r}\left(\Delta^{T} u\right)$, it becomes clear that the large negative velocity increments $\Delta^{T} u$ (that have the same sign as the shear) are more numerous than the large positive ones. This asymmetry grows as the magnitude of the increments increases. At large orders, therefore, both even and odd-order moments are dominated by the negative increments and the asymmetry between odd and even exponents will disappear. This trend is evident from the measured scaling exponents in Fig. 3. We also see that the scaling of the transverse velocity increments in the shear direction is strongly anomalous: the deviation from the Kolmogorov self-similar prediction is much larger than for increments measured in other directions. As a reference we show measured scaling exponents from longitudinal velocity increments $\left|u\left(\boldsymbol{x}+\boldsymbol{r} \boldsymbol{e}_{x}\right)-u(\boldsymbol{x})\right|$, which follow the prediction of the log-Poisson model [11].

A conspicuous feature of the transverse scaling exponents in Fig. 3 is that they tend for large $p$ to a limiting value $\zeta_{p}^{T}=\zeta_{\infty} \approx 2.8$ which is reached at $p \approx 15$. Before interpreting this behavior, we will first argue that it is significant and is not caused by a lack of statistical accuracy of these large order moments.

First, the saturating behavior of the scaling exponent is reproduced in repeated runs of the same experiment, and is not observed in our experiments on homogeneous 
near-isotropic turbulence. A second argument for saturation is directly related to the measured PDF's $P_{r}(\Delta u)$ of the velocity increments $\Delta u$ at separation $r$. Then, a sufficient, but not necessary, condition for saturation of the scaling exponents is that the function $Q_{r}(\Delta u) \equiv$ $\left(r / r_{1}\right)^{-\zeta_{\infty}} P_{r}(\Delta u)$ becomes for large $|\Delta u|$ independent of $r$ for $r$ values inside the inertial range [12]. Using their stretched exponential representation, we will now show that our PDF's actually satisfy this criterion.

All measured transverse PDF's can be represented well by stretched exponentials $P_{r}^{s}(x)=a_{r} e^{-\alpha_{r}|x|^{\beta_{r}}}$, where the parameters $a_{r}, \alpha_{r}$, and $\beta_{r}$ are different for the negative and positive velocity increments. The difference between $P_{r}^{s}$ and the measured $P_{r}$ is insignificant in a $\chi^{2}$ sense [13]. Saturation of the scaling exponents to $\zeta_{\infty}$ then requires that the function $Q_{r}^{s}(\Delta u)=a_{r}\left(r / r_{1}\right)^{-\zeta_{\infty}} e^{-\alpha_{r}|\Delta u|^{\beta_{r}}}$ becomes $r$ independent for large $|\Delta u|$.

In Fig. 4 we show the function $Q_{r}^{s}(\Delta u)$ for several separations $r$ inside the inertial range $30 \leq r / \eta \leq 300$. It is indeed observed that the more probable, negative tails of the probability density functions become independent of $r$ when properly rescaled. This was, of course, already evident from the scaling exponents in Fig. 3 that were computed from precisely these tails. The inset of Fig. 4 shows, on a much expanded scale, that the function $Q_{r}^{s}(x)$ is completely consistent with our data. At very large $\Delta u$,

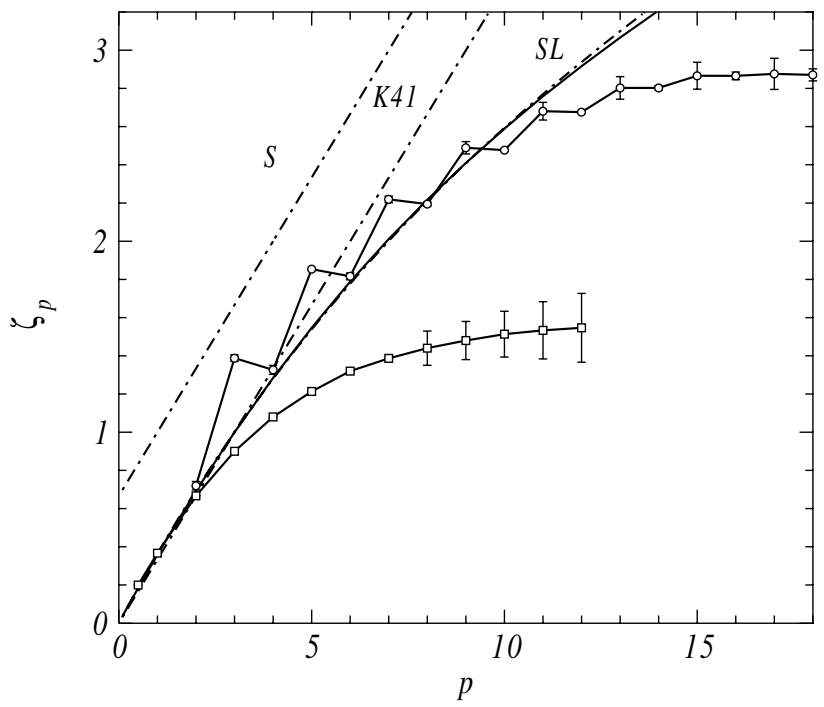

FIG. 3. Scaling exponents of $p$-order moments of velocity increments. Open circles connected by line: $\zeta_{p}$, with the error bars set by the variation of $\zeta_{p}$ in different experiments. Full line: scaling exponents of moments of absolute values of longitudinal velocity increments. Open squares connected by line: scaling exponents for passive scalar fluctuations measured by Moisy et al. [10]. As [10] provides relative values, these exponents have been normalized by setting $\zeta_{2}=2 / 3$. Dashdotted lines (K41): Kolmogorov 1941 prediction [1]; (SL): prediction of log-normal model [11]; (S): shear prediction Eq. (2) of odd order moments. The line of the log normal model obscures the longitudinal measurement. the functions $Q_{r}^{s}(\Delta u)$ for different $r$ start to deviate again, but this is already beyond the $\Delta u$ needed to determine moments of order $p=15$ [14].

Saturation of the scaling exponents $\zeta_{p} \rightarrow \zeta_{\infty}$ points to clifflike structures in the velocity field. This can be readily understood in the framework of the multifractal model, where $\zeta_{\infty}$ would be the codimension of these structures [15]. A similar behavior of the scaling exponents both in numerical simulations [12] and in experiments [10] has been found for the concentration fluctuations of a passive scalar that is advected by a turbulent flow. It has been linked to the well-known "ramp and cliff" structure of the concentration field $[4,12]$. Therefore, the velocity field of turbulence with an imposed large-scale shear and the concentration field in turbulence with a large-scale gradient of the contaminants behave analogously. The difference is that the concentration field is more intermittent than the velocity field so that the saturation of the concentration scaling exponents occurs at lower orders $p$ ( $p \approx 12$ ), as is also illustrated in Fig. 4.

Since we measure the turbulent velocity field with many probes simultaneously, it is possible to search for strong events that extend in the shear $(y)$ direction; this is not possible in point measurements of the velocity field. In our quest for these events, we adopted the simple strategy to look for $n$ velocity profiles $u(x, y)$ which have the largest transversal velocity difference $\left|\Delta u^{T}\right|=$ $|u(x, y+\delta y)-u(x, y)|$ across two closely spaced probes (separation $\delta y / \eta=6$ ), which is also a local maximum in

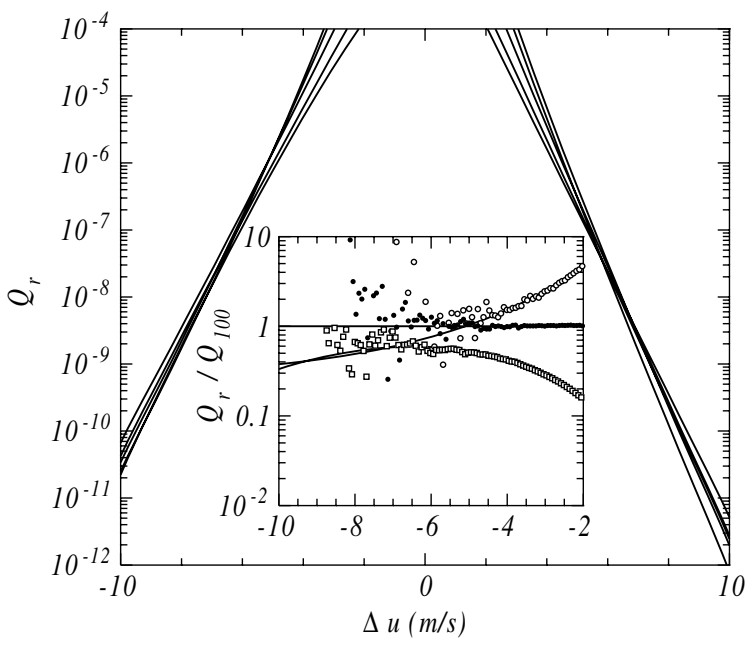

FIG. 4. Full lines: the function $Q_{r}^{s}(\Delta u)=\left(r / r_{1}\right)^{-\zeta_{\infty}} P_{r}^{s}(\Delta u)$, with $r_{1} / \eta=30, r / \eta=30,50,100,190,270$, and $\zeta_{\infty}=2.85$. Inset: Full lines: $Q_{r}^{s}(\Delta u) / Q_{r_{0}}^{s}(\Delta u)$, with $r_{0} / \eta=100$ and $r / \eta$ taken in the inertial range, $r / \eta=30,100$, and 270, respectively. Symbols: $Q_{r}(\Delta u)=\left(r / r_{0}\right)^{-\zeta_{\infty}} P_{r}(\Delta u) / P_{r_{0}}^{s}(\Delta u)$, for $r_{0} / \eta=100$, and $r / \eta=30$ (open circles), $r / \eta=100$ (closed dots), and $r / \eta=270$ (open squares), respectively. Notice that the low probability tails of the PDF are skewed towards the negative velocity increments. 


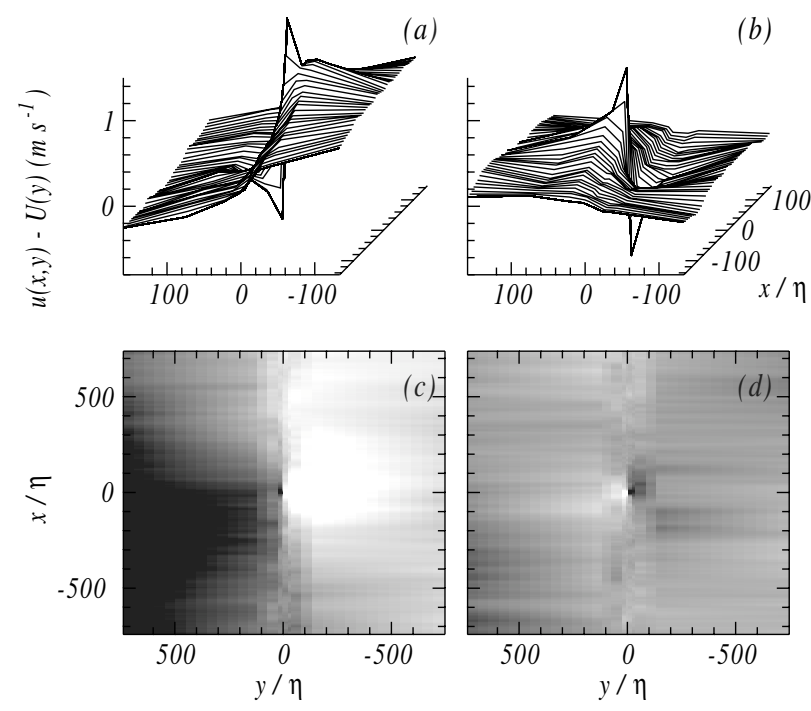

FIG. 5. Average velocity profile of the 256 largest events in a times series of $10^{8}$ velocity line samples. (a) With $\Delta u \equiv$ $u\left(x, y_{6}\right)-u\left(x, y_{5}\right)<0 \quad$ with $y_{6}-y_{5}=1.0 \mathrm{~mm}=9 \eta$. For clarity, the $y$ axis is shown reversed. (b) With $\Delta u>0$. (c) Same as (a), but in gray scale over a larger $(x, y)$ range. It clearly shows the residual large-scale gradient. (d) Same as (c), but $\Delta u>0$.

the $x$ direction. The sign of strong events is favored by the shear, out of $n$ events (e.g., $n=200$ out of $10^{8}$ line samples), $\approx 0.7 n$ have the same (negative) sign as the shear. This is not a simple additive effect; the mean shear gives a mere $\Delta u_{S}=0.1 \mathrm{~ms}^{-1}$ across the viscous-range separation $\delta y$, a factor of 4 smaller than the size fluctuation of the $n$ largest events.

The separate averages shown in Fig. 5 of the positive and negative events were done by choosing the local maximum of $\Delta u$ in the $x$ (streamwise) direction at $x=0$. Most remarkably, the average shape of the positive strongest events is very different from that of the negative ones. While the negative events clearly reveal a clifflike structure of the velocity field, the average positive events are indistinguishable from those found in near-isotropic turbulence and do not carry the imprint of the large-scale shear. As high-order structure functions are determined by the negative events, it can now be understood why the behavior of the scaling exponents in shear turbulence differs from those in (near-)isotropic turbulence.

In conclusion, strong turbulence that is driven by a uniform mean shear at its largest scales shows the imprint of this anisotropy at the small scales. The failure of the return to isotropy is reflected both in the behavior of the scaling exponents and in the occurrence of extremely intermittent structures which have remembered the way in which the turbulent flow is driven. Although the Reynolds number of our experiment $\left(\operatorname{Re}_{\lambda}=630\right)$ is modest, there is a clear inertial range, and we believe that the persistence of anisotropies is a genuine property of strong turbulence, a conclusion that is consistent with [2].

We gratefully acknowledge financial support by the "Nederlandse Organisatie voor Wetenschappelijk Onderzoek (NWO)" and "Stichting Fundamenteel Onderzoek der Materie (FOM)." We are indebted to Gerard Trines, Ad Holten, and Gerald Oerlemans for technical assistance.

[1] A. Kolmogorov, Dokl. Akad. Nauk. 30, 4 (1941); reprinted in Proc. R. Soc. London, Ser. A 434, 9-13 (1991).

[2] X. Shen and Z. Warhaft, Phys. Fluids 12, 2976 (2000).

[3] M. Ferchi and S. Tavoularis, Phys. Fluids 12, 2942 (2000), although restoration of isotropy is concluded, $S_{3}^{T}$ decreased slower with $\mathrm{Re}_{\lambda}$ than expected. A reconciliation with the result of [2] is given by Z. Warhaft and X. Shen, Phys. Fluids 13, 1532 (2001).

[4] A. Pumir and B. I. Shraiman, Phys. Rev. Lett. 75, 3114 (1995).

[5] J. L. Lumley, Phys. Fluids 10, 855 (1967).

[6] The variation of $\left\langle u^{2}\right\rangle^{1 / 2}$ with $y$ is significantly smaller than that reported in [2], which may also explain the different behavior of the scaling exponents in [7].

[7] X. Shen and Z. Warhaft, Phys. Fluids 14, 370 (2002).

[8] S. G. Saddoughi and S. F. Veeravalli, J. Fluid Mech. 268, 333 (1994).

[9] S. Kurien and K. R. Sreenivasan, Phys. Rev. E 62, 2206 (2000) find $\alpha=-2.1$, while Z. Warhaft and X. Shen, Phys. Fluids 14, 2432 (2002) find $\alpha=-2.0$.

[10] F. Moisy, H. Willaime, J. Andersen, and P. Tabeling, Phys. Rev. Lett. 86, 4827 (2001).

[11] Z.-S. She and E. Leveque, Phys. Rev. Lett. 72, 336 (1994). This model is found to accurately parametrize many measurements of the scaling of $\left\langle\left|\Delta^{L} u\right|^{p}\right\rangle$.

[12] A. Celani, A. Lanotte, A. Mazzino, and M. Vergassola, Phys. Rev. Lett. 84, 2385 (2000).

[13] W. van de Water and J. A. Herweijer, J. Fluid Mech. 387, 3 (1999). A stretched exponential description is strictly untenable [see A. Noullez, G. Wallace, W. Lempert, R. B. Miles, and U. Frisch, J. Fluid Mech. 339, 287 (1997)]. The stretching exponent ranges from $\beta_{r}<1$ at small $r$ to $\beta_{r}=2$ at integral-scale separations $r$. For $|\Delta u|$ large enough, small- $r$ velocity increments of a given size $\Delta u$ would then have a larger probability than large- $r$ increments of the same size, which is impossible. Since the small- $r$ PDF's are narrower than the large- $r$ ones, this will occur only at extremely small probability levels and would be observed only at a number of samples that is many orders of magnitude larger than what we used $\left(10^{9}\right)$.

[14] The maximum $\Delta u_{m}$ needed is set roughly by the value where $\Delta u^{15} P_{r}(\Delta u)$ reaches a maximum, which is at $\Delta u_{m}=-3,-5$, and $-8 \mathrm{~ms}^{-1}$ for $r / \eta=30,100$, and 270 , respectively.

[15] U. Frisch, Turbulence (Cambridge University Press, Cambridge, 1995). 